\title{
Sociedad de la información y educación: contextos para la actividad gerencial
}

\author{
Prof. Dr. D. Javier Tarango Ortiz ${ }^{1}$ \\ Prof. M.C. D. Gerardo Ascencio Baca ${ }^{2}$ \\ Prof. M.C. Doña Patricia Murguía Jáquez ${ }^{3}$ \\ Prof. M.C. D. José Refugio Romo González ${ }^{4}$
}

\section{Resumen}

Comprende un análisis de los conceptos básicos sobre la sociedad de la información y sus dimensiones, de lo cual se deriva el abordaje de los ámbitos educativos considerando la propia dimensión que éstos dan al tema aquí estudiado. Pone de manifiesto la importancia de establecer una concordancia entre los propósitos de los sistemas educativos nacionales con los mercados laborales, ya que ambos representan los mecanismos de culturización de sujetos de una forma más ordenada. Finalmente, incluye un listado de herramientas metodológicas por medio de las cuales los individuos, especialmente los relacionados con la ciencia de la información, pueden contribuir de forma directa al desarrollo de la sociedad de la información y otras más, que representan las competencias generales que se deben generalizar y cuya adquisición y aplicación tienen que ver más con las habilidades naturales de los individuos.

Palabras Clave: Herramientas gerenciales. Sociedad de la información. Educación. Comportamiento organizacional.

\section{Resumo}

Compreende uma análise dos conceitos básico sobre a sociedade da informação e suas dimensões, do qual se deriva a abordagem dos âmbitos educativos considerando a própria dimensão que estes dão ao tema aqui estudado. Destaca a importância de estabelecer uma concordância entre os propósitos dos sistemas educativos nacionais com os mercados de trabalho, já que ambos representam os mecanismos de aculturação dos indivíduos de uma

\footnotetext{
1 jtarango@uach.mx, Universidad Autónoma de Chihuahua, Facultad de Filosofía y Letras, Chihuahua, Chih., MÉXICO.

2 gbaca@uach.mx, Universidad Autónoma de Chihuahua, Facultad de Filosofía y Letras, Secretaría de Extensión y Difusión, Chihuahua, Chih., MÉXICO.

${ }^{3}$ pmurguia@uach.mx, Universidad Autónoma de Chihuahua, Facultad de Filosofía y Letras, Chihuahua, Chih., MÉXICO.

${ }^{4}$ jromo@uach.mx, Universidad Autónoma de Chihuahua, Facultad de Filosofía y Letras, Chihuahua, Chih., MÉXICO.
} 
forma mais ordenada. Finalmente, inclui uma lista de ferramentas metodológicas por meio das quais os indivíduos, especialmente os relacionados com a ciência da informação, podem contribuir de forma direta com o desenvolvimento da sociedade da informação e outras mais, que representam as competências gerais que se deve generalizar e cuja aquisição e aplicação têm mais a ver com as habilidades naturais dos indivíduos.

Palavras-chave: Ferramentas gerenciais. Sociedade da informação. Educação. Comportamento organizacional.

\begin{abstract}
The article incluyes an analysis of the Basic concepts of the information society and its dimensions, which comes Fromm the educational environmental, at the same time it takes in consideration the extent included in the mentioned subject. It shows the importance of establishing and agreement between the purposes of the National Education System of Mexico and the labor market. As both represent the mechanisms int the refinement of the individual intellect in a proper order. Finally it includes a list of methodological tools through which the individuals, specifically those who a related with the field of information science who can contribute directly to the growth of the scence of education and those that represent general competences that must be generalized and whose acquisition and application has to do with individual qualities and skills.
\end{abstract}

Keywords: Management tools. Information society. Education. Organizational behavior.

\title{
1. Introducción.
}

La literatura publicada recientemente, tanto en el ámbito de las ciencias de la información como en cualquier disciplina de las organizaciones, enfatizan la tendencia marcada hacia el desarrollo de procesos de trabajo tomando como referencia la formación y el desarrollo de sujetos capaces de enfrentar la sociedad de la información y del conocimiento. Aunque existe en ocasiones baja definición o diferenciación conceptual de los términos utilizados, se logra entender que tanto los ámbitos educativos como los laborales están demandando la posibilidad de formar sujetos con nuevas perspectivas para enfrentar los retos que se le demanden. 
Para cubrir con las expectativas del título planteado en este trabajo, qué básicamente se centra en analizar de cuatro conceptos fundamentales: herramientas gerenciales, sociedad de la información, educación y ámbitos empresariales, se procederá a abordarlos desde diversas perspectivas y en distintos momentos tomando en consideración el siguiente orden de estudio: primero, definir y entender la concepción de sociedad de la información especialmente tomando como referencia distintas tendencias, así como factores que limitan su desarrollo como la brecha digital y el desnivel de conocimiento; segundo, conceptuar la forma como los ámbitos educativos entienden y comprenden tales enfoques básicos para identificar el producto educativo del futuro; tercero, igualmente diferenciar la forma como las organizaciones consideran a un sujeto culturizado en esta tendencia; y finalmente, enlistar aquellas herramientas generales que se consideran pertinentes para, de forma concreta, enfocar los procesos hacia tales iniciativas.

Pareciera que un punto de partida es la confusión generalizada en que viven la mayoría de los sujetos en torno al concepto de sociedad de la información, los cuales quizá conciban de forma distinta esta perspectiva y solamente se asocien a ámbitos del capitalismo, industrialismo, totalitarismo o democracia liberal. $\mathrm{O}$ bien imaginarse, sin darle importancia a que este tipo de conceptos son únicamente ocurrencias históricas que nos tocó vivir y que realmente son eventos generales de los cambios sociales, económicos y políticos de la sociedad actual.

Autores como Webster (2002) aborda la dificultad que existe en definir el concepto de sociedad de la información, dando a conocer que es algo que todos entendemos pero que puede comprender demasiados aspectos, tales como las formas de producción económica, nuevas formas de interacción social, procesos de innovación o los tres enfoques al mismo tiempo. Bajo este estilo se presentan la mayoría de las definiciones proporcionadas en documentos tipo ensayo, por lo que se consideró más pertinente recurrir a términos enciclopédicos, tales como los presentados por López Yepes (2004) en su Diccionario Enciclopédico de las Ciencias de la Documentación quien define a la sociedad de la información como "sociedad caracterizada por la facilidad para producir y recibir mensajes, por las crecientes necesidades sociales de información y por el desarrollo incesante de las nuevas tecnologías de la información”.

El autor antes citado hace además una diferenciación con la sociedad de la documentación en donde considera que el ámbito imperante se caracteriza por el creciente desarrollo de la función documental al producirse muchos documentos consecuencia de numerosos mensajes vehiculazos en soporte físico. Sin embargo, el concepto de información 
lo considera como un proceso generalizado que ofrece uno o varios contenidos, los cuales pueden estar plasmados en documentos o bien en elementos no tangibles tales como la comunicación verbal, el registro individual de experiencias o cualquier otro medio de transmisión de datos de un sujeto a otro.

Si bien a la información se le concibe como un proceso que conlleva contenidos, los cuales como elementos tangibles, pueden identificarse como documentos y otros no tangibles conceptualizados como conocimiento, que están asociados a la representación mental del mundo real o de sus estados potenciales conocidos como estructura de conocimiento y los mecanismos para su obtención y aplicación nombrados dinámica del conocimiento (López Yepes, 2004).

Se logra inferir entonces, que aunque diversos autores usen de forma separada los descriptores de sociedad de la información, sociedad del conocimiento o sociedad de la información y el conocimiento, todos representan la posibilidad del sujeto de asimilar de forma correcta y mesurada, tanto lo tangible o lo intangible de diversos conjuntos de datos lógica o ilógicamente ordenados para aplicarlos en la solución de problemas concretos. Por tanto, el sujeto que la sociedad demanda deberá ser aquel que para sí mismo y los demás muestra la habilidad para diseminar información en cantidades y momentos correctos y cuyo resultado sea la solución coherente de situaciones cotidianas. Por lo que el concepto de sociedad de la información se puede inferir como más incluyente ya que comprende a la información misma como proceso tangible logrado a través de documentos y a los procesos intangibles entendidos como conocimiento, los cuales a su vez pueden llegar a registrarse y funcionar en otras dimensiones.

Dada la confusión conceptual, pero que aparentemente se lleva a creer que finalmente se comprende el concepto, se considera mejor diferenciar los ámbitos en los cuales se manifiesta la sociedad de la información, y aunque Webster (2002) no logra definir el concepto de forma concreta, sí logra incluir una serie de ideas de la sociedad de la información que distingue cinco conceptos vinculados con los ámbitos en los cuales se manifiesta de forma dinámica ya que su presencia no es particular de escenarios exclusivos, Las cinco ideas son:

a. Concepción Tecnológica. Básicamente vinculada a la innovación, la cual toma mayor forma en los años 70's siendo el indicador más tangible de la sociedad de la información. Se concibe aquí la participación y conocimiento del sujeto en el uso y 
manejo de las tecnologías de la información y las comunicaciones (TIC) como herramientas comunes a la actividad laboral o social del sujeto.

b. Concepción Económica. Este enfoque ve a la actividad informacional desde la perspectiva de su contribución al crecimiento del producto interno bruto (PIB). En este aspecto se asume la presencia de la investigación y desarrollo (I+D) como una forma fundamental de demostrar la actividad informacional.

c. Concepción Ocupacional. Este enfoque es el más favorecido por los sociólogos y es básicamente asociada con el trabajo de Bell (1973) que es un teórico que ha estudiado lo que él llama la "sociedad post-industrial", misma que virtualmente se considera un sinónimo de la sociedad de la información. Esta concepción esta directamente vinculada con la estructura organizacional como un patrón de cambio observado.

d. Concepción Espacial. Apreciada tanto por sociólogos como por economistas tiene como indicador distintivo en la medición del espacio geográfico, considera redes de información que conectan lugares y en consecuencia tienen un efecto importante en la organización del tiempo y espacio.

e. Concepción Cultural. Esta última alternativa es la más fácil de reconocer, pero la menos medible. Es la información que se encuentra en la circulación social y tiene un vínculo muy directo con la medición de la cultura organizacional o social imperante en un determinado momento o circunstancia.

Según la clasificación anterior sobre los ámbitos de la sociedad de la información se logran identificar los instrumentos o herramientas que la sustentan, la repercusión en los aspectos económicos, la identificación de los sujetos sociales que la practican, su distribución en el contexto geográfico y macrosocial, y la forma como se manifiesta a través del comportamiento cotidiano en los sujetos que la practican. En relación a esta tipología taxonómica de los ámbitos de la sociedad de la información se desarrollará el análisis de los contenidos que se incluyen en el resto del ensayo.

Sin embargo, es importante no dejar al margen elementos como la brecha digital y el desnivel de conocimiento. Esto porque el paso de una sociedad industrial a una sociedad basada en la información constituye un avance fundamental en el desarrollo actual de las naciones. La revolución de la información que actualmente se manifiesta, incide en la forma de vivir, aprender y trabajar de las personas y en el modo en que los gobiernos interactúan con la sociedad civil. La información, transformada en conocimiento es una herramienta poderosa 
y ahora, más que nunca, relevante en la evolución económica y cultural de cualquier sociedad (ONU, OIT, 2005).

La libre circulación de información e ideas, premisa fundamental de cualquier democracia, ha desencadenado un crecimiento radical de los conocimientos y de sus múltiples nuevas aplicaciones. Como resultado, se están transformando las estructuras y relaciones económicas, culturales y sociales. Sin embargo, una inmensa mayoría de la población de los países que se encuentran en la fase de incorporación de las nuevas tecnologías y en proceso de integrarse a la globalización, permanece al margen de esta revolución. Esta brecha digital y el desnivel de conocimiento amenazan con agrandar la disparidad ya existente en materia de desarrollo entre ricos y pobres, dentro de las naciones y entre éstas. No se podrá conseguir un beneficio generalizado de tal proceso, a menos que se tomen medidas para acercar y poner a disposición de las mayorías los medios que permitan una participación plena en la emergente sociedad del conocimiento sustentada en la información (Ascencio, 2006).

\section{Sociedad de la información y educación}

Dos ámbitos fundamentales que influyen en el desarrollo de la sociedad de la información en los contextos de cualquier país son especialmente: los educativos y los laborales. El primero con mayor control en su implantación ya que comprende a los sujetos que forman una sociedad en diversas etapas de su vida a través de un sistema educativo nacional; el segundo, menos controlado pero dependiente del anterior en razón de que se puede suponer que gran parte de sus insumos humanos estarán relacionados con un patrón de formación, independientemente de que se responsabilice además de aquellos sujetos que no fueron incluidos en la educación formal.

Analizando primero el ámbito educativo, para posteriormente el laboral, se observa que dada la diversidad de sistemas nacionales de educación, uno por país y en ocasiones divididos en públicos y privados dentro de una misma región, además del conocimiento y origen de los autores de este trabajo, se presenta a continuación un breve análisis de la forma como el sistema educativo nacional de México describe y concibe a la sociedad de la información a través de su Programa Nacional de Educación, 2001-2006 (2000) en sus diversos niveles educativos y analizado en otros foros relacionados con las ciencia bibliotecaria y de la información (Tarango, 2004a y 2004b). 
En este documento se entiende que la educación para el futuro de México, representada en todos los niveles y modalidades, debe cubrir la necesidad de formar personas con características precisas y cualidades diversas para afrontar la sociedad de la información, incluyendo en este proceso las siguientes consideraciones:

a) El dominio de aspectos que determinan la generación, apropiación y uso del conocimiento;

b) Capacidad para trabajar en ambientes de tecnología de información y comunicación;

c) Deseos de propiciar y facilitar el aprendizaje;

d) Capacidad para despertar el interés, la motivación y el gusto por aprender;

e) Disponibilidad para aprender por cuenta propia y a través de la interacción con otros;

f) Habilidad para estimular la curiosidad, la creatividad y el análisis;

g) Aptitudes para fomentar la comunicación interpersonal y trabajo en equipo;

h) Imaginación para identificar y aprovechar oportunidades diversas de aprendizaje;

i) Autoridad moral para trasmitir valores a través del ejemplo.

Los cambios en los procesos de aprendizaje suponen al aula no como el único ambiente educativo para el aprendizaje, el profesor se convierte en un facilitador del proceso, la asimilación de las tecnologías informáticas serán comunes en estudiantes y maestros, así como la lucha de que la generación y aplicación de conocimientos no se limite a grupos e instituciones dominantes, sino a la generalidad de instituciones de educación en el país, tanto públicas como privadas.

Las dimensiones educativas que el Programa Nacional de Educación 2001-2006 de México propone son tres: El microsistémico considerada como la unidad mínima de un sistema educativo en donde cada institución debe ser capaz de diagnosticar sus problemas y de planear las formas de resolverlos; el intermedio, consiste en la profesionalización de cuadros dentro de un ámbito particular para funcionar en un ámbito más general; el macrosistémico, el cual se refiere a la implantación de políticas nacionales, en este sentido están los cambios de currículo y el desarrollo de materiales innovadores generalizables a poblaciones mayores; por tanto, se percibe que la sociedad de la información y del conocimiento deberá estar presente en todas las dimensiones, tanto institucionales como sociales. 
Puede concluirse que en el caso de México existe una baja definición de la concepción de sociedad de la información en relación con el ámbito educativo como medio coherente para trasmitirla como cultura general de un conglomerado nacional. Sin embargo cabe resaltar que da importancia al ámbito tecnológico al considerar que el sujeto se debe relacionar de forma natural con ello. En cuanto al ámbito espacial, lo define desde la perspectiva que pretende llegar a todos los niveles educativos que forman el sistema educativo nacional, dando mayor importancia a aspectos culturales en cuanto a la generación y agrupación de conocimiento y la interrelación con los demás sujetos que compongan la estructura ocupacional del participante. De forma muy sutil implica la presencia del ámbito económico cuando promueve la creatividad como rasgo cultural como fuente para propiciar la innovación.

Aunque el propósito inicial no es analizar las condiciones que guardan los lineamientos educativos de un país en particular en relación con la sociedad de la información, el análisis antes incluido sí representa la preocupación que todo modelo educativo debe conllevar implícito en la definición del producto que deberá ofrecer a la sociedad en razón de la tendencia y conceptualización imperante en el mercado en relación a la forma como se deberá comportar el sujeto en escenarios posteriores. Siendo el sistema educativo nacional un medio relativamente fácil de manejar, no debe de pasar desapercibido como un instrumento para fomentar las habilidades gerenciales que los mercados laborales demanden posteriormente.

\section{Sociedad de la información y ambientes laborales.}

Frecuentemente se cuestiona si los ámbitos educativos están trabajando en coordinación con los ámbitos laborales o si existe un desprendimiento permanente en donde cada cual considera lo que espera de los sujetos participantes en la sociedad de la información. Se puede suponer que sí existe tal identificación de forma que como se registra que debe prepararse al sujeto será como el mercado laboral demanda ese recurso humano.

Un análisis generalizado de la forma como las organizaciones conciben a un sujeto competente, se resume en la propuesta de Meister (2000) quien indica que las organizaciones reciben múltiples personas que ingresan a sus actividades laborales pero que no necesariamente han recibido de sistemas educativos formales el entrenamiento para afrontar 
sus requerimientos, a lo que propone desarrollar modelos educativos de aprendizaje organizacional conceptualizados como Universidades Corporativas.

Las organizaciones más visionarias están adoptando el cambio de generar la educación continua tomando como referencia su propia infraestructura. Éstas organizaciones han encontrado en las universidades corporativas una nueva estrategia para combatir el déficit de talento que existe en el mercado laboral y formar personal a la "medida exacta" de sus necesidades. Su objetivo principal es el de capacitar y actualizar a todo el personal en sus propias instalaciones, a partir de programas diseñados por la misma compañía y así desarrollar el talento o capital intelectual que la firma necesita para obtener ventajas competitivas.

Las organizaciones están buscando dar nuevas oportunidades de aprendizaje para enfrentarse a los mercados globales, desarrollar relaciones profundas con sus clientes y proponer el diseño de las organizaciones del futuro. Además, en las universidades corporativas los participantes reciben títulos que pueden hacer valer en la propia organización, tal como si fuera una certificación de calidad, que los faculta para una carrera ascendente dentro de la firma.

La forma como trabaja el modelo de Universidades Corporativas se manifiesta en las siguientes características:

a) Provee oportunidades de aprendizaje que soporte las soluciones de negocios de problemas críticos de la organización.

b) Considera al Modelo Educativo un proceso más que un lugar de aprendizaje.

c) Diseña un currículum que incorpore: Identificación organizacional, Conocimientos y Competencias.

d) Entrena en la cadena del valor, incluyendo: clientes, distribuidores, proveedores e instituciones educativas externas que provean personas $y$ programas en un futuro.

e) Evolución del entrenamiento por instructor a formatos múltiples de ofrecimiento del aprendizaje.

f) Genera líderes que se involucren en facilitar el aprendizaje a otros.

g) Mueve a la organización del modelo corporativo al auto-funcionamiento de unidades de negocios.

h) Asume un enfoque global en el desarrollo de soluciones de aprendizaje.

i) Crea un sistema de medición para evaluar todos los elementos de aprendizaje, tanto internos como externos. 
j) Utiliza el Modelo Educativo como una ventaja competitiva y entrar en nuevos mercados.

Los mercados laborales son menos explícitos en comparación con los ambientes educativos en la forma como conciben a un sujeto competente ante la sociedad de la información y de acuerdo a Meister (2000), las expectativas organizaciones definen a un sujeto capaz de enfrentar tales situaciones tomando como referencia que demuestre capacidad para:
a) Aprender a aprender
b) Comunicarse y colaborar
c) Pensar creativamente y solucionar problemas
d) Usar la tecnología
e) Globalizar los negocios
f) Desarrollo de liderazgo
g) Auto-administración de carrera y plan de vida

Aparte de la estructura de competencias laborales antes descritas, en algunos contextos empresariales ha llegado a ser común el uso de herramientas de medición de la efectividad de los sujetos considerando competente aquellos que observen: capacidad administrativa (administración del tiempo, definición de metas y objetivos, y planeación y programación), capacidad comunicativa (escuchar y organizar información, proporcionar información clara y obtener información específica), capacidad de supervisión (entrenando y delegando, evaluación del desempeño y corrigiendo y aconsejando) y capacidad cognitiva (identificación y solución de problemas, toma de decisiones y pensamiento objetivo y analítico), todos ellos vinculados con el uso constante de las tecnologías de la información y las comunicaciones (Training House, 2002).

Lejos de encontrar diferencias significativas, lo cierto es que tanto la concepción de los sistemas educativos como la de los medios laborales, en relación con la sociedad de la información, ofrecen alternativas relativamente similares en cuanto a la forma como relacionan las características que globalmente debe demostrar un sujeto para ser considerado competitivo. Finalmente se puede percibir que el problema no está en la identificación de tales sellos distintivos sino en la forma en que lo adquiera el sujeto a través de la formación educativa o por medio de la experiencia laboral. 


\section{Propuestas de herramientas gerenciales}

Las herramientas gerenciales para desarrollar las actividades que la sociedad de la información demanda, en cualquier ámbito organizacional, pueden dividirse en dos tipos: 1). Las de carácter metodológico (vinculadas regularmente a la disciplina de las ciencias de la información, en donde se generan mecanismos para el procesamiento de información y conocimiento de forma tangible); y 2). Las de carácter inherente (aquellas que están presentes en cualquier proceso de trabajo sin diferenciar la disciplina a la que pertenecen, sino más bien, que son parte de los procesos que conllevan a contribuir a la sociedad de la información como una cultura).

En cuanto a las herramientas gerenciales de carácter metodológico, aparte de las ya conocidas como medios de soporte tales como catalogación, clasificación, indización, etc., y el uso necesario de las tecnologías de la información y la comunicación (TIC), están las que denotan el registro de conocimiento para convertirlo en información documental y viceversa, tales como:

a) Documentación de procesos de operación. Referida al registro documental de las formas de trabajo de los sujetos inmersos en las organizaciones, comprende el rediseño y la innovación de aquellos procesos que lo requieran.

b) Generación de productos documentales y servicios de información. Trabajar en pro de la dependencia informativa buscando mecanismos de producción de información propia. Aquí se considera pertinente obtener experiencia en el uso de manuales de estilo para reproducir documentos tipo manuscritos, editados o ambos.

c) Mapeo de información estratégica. Registro de las fuentes de información estratégica que utilizan las entidades organizacionales de tal forma que no se concentren en espacios específicos sino en los campos de acción del sujeto que los utiliza.

d) Administración y resguardo de información inactiva generada por la misma organización. Implantación de procedimientos de resguardo de información legal, administrativa, fiscal, histórica, etc. que la organización ha generado para su funcionamiento y que es necesario conservar de acuerdo a las reglamentaciones regionales en donde se encuentre establecida la entidad productiva o de servicios. 
e) Desarrollo de modelos de aprendizaje organizacional. Creación de formas adecuadas de compartición y adquisición de conocimiento tomando como referencia que el sujeto participante es un adulto y su forma como aprende es distinta a los estudiantes tradicionalmente inmersos en ambientes escolares.

f) Acceso a fuentes y servicios de información generados por otros. Localización y uso de los recursos informativos creados por otras instancias, los cuales se demandan para conocer los avances generados en el ambiente exterior.

Las herramientas gerenciales de carácter inherente, las cuales no pueden estar ajenas o separadas a las de carácter metodológico, pueden ser tan amplias como la competencia del sujeto lo permita, pero fundamentalmente se consideran los siguientes rasgos que pueden servir de base para sustentar mejor los procesos que demanda la sociedad de la información. Estas son:

a) Capacidad diagnóstica de conocimiento existente. Tiwana (2000) propone como elemento inicial, partir de un diagnóstico que registre lo que se conoce como un análisis de la infraestructura existente, en donde el empleado manifieste aquellos conocimientos (tácitos o explícitos) que posee y le sirven para funcionar en su posición, también se consideran aquellos conocimientos que son necesarios a su puesto y que no posee, esto según su propia visión y la de otras instancias pertinentes al caso (jefes superiores, personal en posiciones similares, consultores externos, etc.). Se recomienda hacer una alineación de las necesidades de conocimiento con las estrategias de la organización, llegando a definirse mapas de conocimiento tomando como referencia niveles de conocimiento (central, avanzado e innovador).

b) Capacidad de registro de conocimiento tácito-explícito. Definir el conocimiento tácito, según Wong y Radcliffe (2000), es relativamente simple para describirlo en términos abstractos, pero difícil cuando se trata de dar un concepto concreto, considerando como aquel conocimiento almacenado o expresado de forma implícita entendida de forma inferida, así como aquel cuerpo de conceptos que se puede trasmitir de individuo a individuo y es considerado como un conocimiento en acción trasmitido entre iguales (Bergquist, Jungberg y Lundh-Snis, 2001), además, que la mayoría de los conocimientos ordinariamente son basados en la experiencia. En contraste, el 
conocimiento conocido como explícito, es aquel que está claramente articulado, comunicado abiertamente y regularmente asentado en documentos; este tipo de conocimiento es factible ser trasmitido de una persona a otra $u$ otras de manera formal, en lenguaje sistemático o a través de códigos. En ambos casos (Koch, 2002), lo importante es el establecimiento de efectivas interfaces entre los miembros de las organizaciones.

c) Desarrollo de escenarios de compartición de conocimiento. Surge (Gareth y Caldwell, 2002), a través del trabajo colaborativo que consiste en el intercambio de información desde una diversidad de perspectivas en una situación particular. La posibilidad de desarrollar reuniones cara a cara o usando tecnología es como se describe el modelo de colaboración humana en donde pueden experimentarse las siguientes versiones: persona a persona (individuo o grupo), persona a tecnología de la información y tecnología de la información a persona.

d) Establecimiento de procesos de comunicación efectiva. Este aspecto resulta fundamental para distintas facetas de los procesos ya que la interrelación de los distintos individuos que participan en él se hace necesaria a la actividad aquí propuesta. La comunicación es vista aquí desde dos perspectivas: la primera, referida la interrelación de sus actores de forma personal para lograr la transferencia de conocimiento entre individuos; y la segunda, relativa a la intervención de tecnología para contribuir al proceso mismo (Roberts, 2000; Vaux, 2001; Varey, Wood-Harper y Wood, 2002; Wahl, 2003). En el caso de la interrelación entre personas ésta se facilita sí se consideran algunos aspectos de la medición de la cultura organizacional, al que según Hofstede (1991) es llamado por él mismo como individualismo-colectivismo, en donde los participantes poseen más capacidad de trabajo individual o solitario contra los que tienen capacidad de trabajar en equipo o comunidades. En lo relativo a la participación de la tecnología dentro de la transferencia de conocimiento, algunos teóricos como Wahl (2003) opinan sobre la importancia de la comunicación técnica dentro de organizaciones que aprenden tomando como referencia tres áreas de intervención: 1) la forma como se crea o adquiere, disemina y usa el conocimiento, 2) la forma como se usa el conocimiento efectivamente dentro de las organizaciones y 3) la forma como se desarrollan patrones similares de comunicación. 
e) Capacidad de liderazgo. Siempre se dice que los líderes de las organizaciones son las personas que más comprometidas deben estar con los procesos de las organizaciones y que la creencia que ellos tengan sobre estas iniciativas se verán reflejadas en sus resultados en todos los niveles de la estructura. Hailey y James (2002) desarrollaron un artículo titulado "Learning Leaders: The Key to Learning Organisations" en donde analizan la importancia que se tiene en una organización en los procesos de aprendizaje cuando sus líderes se involucran de forma significativa, considerando aspectos tales como: el involucramiento de todos los niveles de una organización o de una sociedad, el aprendizaje a través de la práctica, el aprendizaje a través de la participación del staff, la aceptación de adquirir conocimiento por medio de actores externos, la participación en eventos formales de entrenamiento (como alumnos y como instructores), el involucramiento en el desarrollo de investigaciones y la participación en el establecimiento de procesos de monitoreo y evaluación.

f) Influencia en las expectativas de las organizaciones. Aunque regularmente se habla de que las entidades productoras de bienes y servicios deberían estar orientadas al diseño de esquemas de "organizaciones que aprenden" (Hatchuel, Le Masson y Weil, 2002), para que un proceso de gestión de conocimiento suceda o sea aceptado por una organización como un proceso válido de trabajo, se requieren además involucrarse múltiples factores, los cuales, según Holsapple y Joshi (2002) los clasifica como los seis tipos de recursos: 1) Conocimiento de los participantes (los cuales pueden ser recursos humanos como los empleados y/o recursos materiales como pudieran ser los sistemas computacionales, 2) Cultura que incluye los valores, principios, normas (escritas y no escritas), supuestos, creencias y procedimientos de las organizaciones mismas, 3) Infraestructura, la cual más que los aspectos contables se refiere a la definición de roles, la relación entre esos roles y las regulaciones, 4) Artefactos del conocimiento, considerados como los aspectos que representan el conocimiento como pueden ser: videos para entrenamiento, libros, patentes y productos, 5) Propósito, considerado como la razón de existir de una organización, tales como su misión, visión, metas y objetivos, y 6) Estrategias, como intenciones de logro organizacional. 
g) Contribución a la globalización de la cooperación en conocimiento. La cooperación entre personas, organizaciones y países puede estar matizada con múltiples modalidades de interrelación, ya sea de forma unilateral, bilateral y multilateral, esto con fines de ayuda de una entidad fortalecida a una débil, de cooperación entre iguales circunstancias, entre otras variantes. En el caso de la compartición de conocimiento, experiencias y prácticas quizá una de las formas más comunes ha sido a través de la interrelación de documentos generados en distintas entidades y utilizados como referencia en otras, sin negar la intervención de medios electrónicos como forma de comunicación más efectiva.

h) Legitimación de procesos. Representan la posibilidad de considerar que el común de la gente acepte como válida su existencia, viendo su pertenencia dentro de las estructuras organizacionales no solo como acciones permanentes, sino inclusive, como pertenecientes a un departamento en particular o ser un departamento en sí. Esto significa, a opinión de Bergquist, Ljungberg y LundhSnis (2001) que cuando algo es razonable para algunas personas y no lo es para otras, seguramente los procesos no están legitimados y por tanto los nuevos conocimientos en las organizaciones no están certificados ni institucionalizados. Cuando se ha reunido un grupo de personas a compartir conocimientos o prácticas que se inclinan a un nuevo conocimiento, pueden existir diferentes puntos de vista, incluso de aceptación o rechazo, sin embargo, desde que los participantes están en un grupo de compartición están involucrados en el grupo mismo, manifestando su acuerdo o desacuerdo. Algunos otros autores como Ruef (1998) y Arndt (1999) han hecho estudios muy particulares respecto a la legitimación de la cultura organizacional y del conocimiento en organizaciones relacionadas con la salud y han considerado que dadas las características tan particulares de los hospitales, y del comportamiento de los miembros de las instituciones médicas, los individuos buscan siempre que lo que se entiende por cultura se legitime, esto es, se oficialice. Además, Young (2000), indica que para que ese proceso suceda, es necesario usar seis niveles en el manejo organizacional: 1) Estudiar lo visible, audible y las manifestaciones tangibles, 2) Registrar los supuestos básicos, 3) Definir los valores compartidos, 4) Identificar las acciones ocasionales, 5) Entender los modos de conflicto, y 6) Entender los niveles de manejo de 
conflicto. Si bien en organizaciones distintas, la legitimación de un proceso resulte diferente, es sorprendente como en las áreas de salud sea parte de su cultura, incluso, Bergquist, Ljungberg y Lundh-Snis (2001) citan como ejemplo a la industria farmacéutica, caracterizado por un trabajo intenso enfocado hacia el conocimiento, al grado que se extiende a su reconocimiento a través de las regulaciones gubernamentales y es en estos sectores en donde más fácilmente se puede lograr la compartición entre iguales, siendo más fácil la compartición tipo explícita que tácita.

\section{Conclusiones.}

El análisis antes presentado comprende el reconocimiento de los sistemas educativos nacionales como herramientas fundamentales para el desarrollo de procesos de culturización masiva de sujetos que entiendan y apliquen los lineamientos de la sociedad de la información, proponiéndose resultados a largo plazo dado que el aprendizaje social se propicia por generaciones.

El punto de partida de la forma como conceptualiza la sociedad de la información en los sistemas educativos nacionales tiene su fundamento en el diagnóstico que se haya hecho de las expectativas de los mercados laborales, entendido como el mercado de oportunidad de trabajo en donde el sujeto podrá manifestar los rasgos adquiridos a través de sistemas formales de aprendizaje. No se debe olvidar que este sector tiene sus propias concepciones, las cuales se supone están alineadas a las educativas con fines de reafirmar rasgos o enriquecerlos.

Dado que los perfiles de los sujetos que enfrentarán los retos de la sociedad de la información no son limitativos de las ciencias de la información sino de cualquier disciplina, primero se reconocen aquellas metodologías propias de los profesionales de la información como medios de desarrollar procesos específicos de gestión, pero fundamentalmente se da mayor importancia a las herramientas básicas que debe observar todo individuo al involucrarse en este tipo de acciones.

Aunque el articulo fundamenta, como punto de partida, a los sistemas educativos como elemento clave en el desarrollo de un proceso de culturización, no se debe olvidar la importancia de considerar mecanismos que envuelvan de manera ordenada a todos los individuos que componen la sociedad y que no necesariamente estuvieron el tiempo suficiente 
inmersos en procesos de educación formal sino más bien sustentados en la experiencia de vida que les tocó experimentar. Por lo tanto, estos procesos basados en la experiencia de vida más que en la educación formal, son sin duda alguna una verdadera alternativa, que bien analizada, documentada y difundida, permite enfrentar junto con la educación formal, la brecha digital y el desnivel de conocimiento. Sirve de apoyo para disminuir la distancia entre info-ricos e infopobres, y que permita el desarrollo de sistemas distribuidos sobre centrales, de sistemas abiertos sobre cerrados.

\section{Referencias bibliográficas.}

Arndt, Margarete. (2000). Presenting Structural Innovations in an Institutional Environmental: Hospitals' Use of Impressions Management. En: Administrative Science Quarterly. September.

Ascencio, Gerardo. (2006). Bibliotecas, Centros de Información y Medios de

Comunicación en la Sociedad de la información. (Documento mecanografiado)

Bell, Daniel. (1973). The Coming of Post-Industrialism Society: A Venture in Social Forecasting. Harmondsworth: Penguin.

Bergquist, Magnus, Jan Ljungberg, Lundh-Snis, Ulrika. (2001). Practicing Peer Review in Organizations: A Qualifier for Knowledge Dissemination and Legitimization. En Journal of Information Technology (16). pp. 99-112.

Garett, Sandra y Barrett Caldwell. (2002). Describing Functional Requirements for Knowledge Sharing Communities. En: Behavior \& Information Technology (Vol. 21, no. 5). pp. 359-364.

Hailey, John y Rick James. (2002). Learning Leaders: The Key to Learning Organisations. En: Development in Practice (Vol. 12, No. 3 \& 4, August). pp. 398-408.

Hatchuel, Armand, Pascual Le Masson y Benoît Weil. (2002). From Knowledge Management to Design-Oriented Organisations. Malden, MA: Blackwell Publishers.

Hofstede, Geelt (1991). Cultures and Organizations. London: McGraw Hill. 
Koch, Michael. (2002). Requirements for Community Support Systems -Modularization, Integration and Ubiquitous User Interfaces. En: Behaviour \& Information Technology (Vol. 21, No. 5).

López Yepes, José, Editor. (2004). Diccionario Enciclopédico de las Ciencias de la Documentación. Madrid: Síntesis.

Meister, J. (2000). Corporate Universities: lessons in building a world-class work force. New York: McGraw Hill.

ONU y UIT. Cumbre Mundial Sobre la Sociedad de la Información. Ginebra 2003-

Túnez 2005. (En Línea) Diciembre de 2003. http://www.itu.int/wsis/basic/about-es.html

Roberts, Joanne. (2000). From Know-How to Show-How?: Questioning the Role of Information and Communication Technologies in Knowledge Transfer. En Technology Analysis \& Strategic Management (Vol. 12, No. 4). pp. 429-443.

Ruef, Martin. (1998). A Multidimensional Model of Organizational Legitimacy: Hospital Survival in Changing Institutional Environmental. En Administrative Science Quarterly. December.

Secretaria de Educación Pública, México. (2000). Plan Nacional de Educación 2001-2006. México: SEP.

Tarango, Javier. (2004a). Educación y sociedad de la información: legitimación necesaria, vínculo obligado. Ponencia presentada en INTERFACES: Foro Internacional sobre Tecnologías de la Información / Universidad de Colima-UNESCO, Colima, Col., México, Noviembre 22-26, 2004.

Tarango, Javier. (2004). Legitimación de la sociedad del conocimiento en el contexto educativo mexicano. Ponencia presentada en las XXXV Jornadas Mexicanas de Biblioteconomía/ Asociación Mexicana de Bibliotecarios, A.C., Cancún, Quintana Roo, México, 11-14 de mayo de 2004.

Tiwana, Amrit. (2000). The Knowledge Management Toolkit: Practical Techniques for Building Knowledge Management Systems. Upper Saddle River, NJ: Prentice Hall.

Training House. (2002). MAP: Examen de Efectividad Gerencial. México: TH.

Varey, Richard J., Trevor Wood-Harper, Bob Wood (2002). A Theoretical Review of Management and Information System Using a Critical Communications Theory. En: Journal of Information Technology (17). pp. 229-239. 
Vaux, Janet. (2001). From Expert Systems to Knowledge-based Companies: How the AI Industry Negotiated a Market for Knowledge. En: Social Epistemology (Vol. 15, No. 3).

Wahl, Scott. (2003). Learning at Work: The Role of Technical Communication in Organizational Learning. En: Technical Communication (Vol. 50, No. 2, May). pp. 247-258.

Webster, F. (2002). Theories of the Information Society. $2^{\text {nd }}$ Ed. New York: Routledge.

Young, Christopher P. (2000). Tacking Stock in Our Progress on Individualism-Collectivism: 100 years of Solidarity and Community. En: Journal of Management (May-June).

Wong, W.L. y D.F. Radcliffe. (2000). the Tacit Nature of Design Knowledge. En: Technology Analysis \& Strategic Management (Vol. 12, No. 4). pp. 493-512. 